\title{
Numerical simulation of aerodynamics of a high-efficiency orthogonal balanced blade of a wind generator
}

\author{
Valeriy Prokopiev", Irina Lantsova and Andrey Proskurin \\ Moscow State University of Civil Engineering, Yaroslavskoe shosse, 26, Moscow, 129337, Russia
}

\begin{abstract}
The results of verification of numerical modeling of a rotating blade of a wind generator with a nonstationary turbulent flow around in a two-dimensional formulation are given. A design free from the drawbacks of a single-blade orthogonal high-efficiency turbine is considered (US Patent Victor Lyatkher, US 8007235 B1, August 30, 2011, RF Patent 2426911 C1).
\end{abstract}

\section{Introduction}

The aim of the work is the verification of the technique of numerical simulation of a rotating blade under the influence of aerodynamic loading with a nonstationary flow around a turbulent flow in a two-dimensional formulation.

\section{Problem statement}

In a single-blade orthogonal high-efficiency turbine (US patent Victor Lyatkher, US 8007235 B1, August 30, 2011, RF patent $2426911 \mathrm{C} 1$ ), the centrifugal forces and moments of these forces are balanced, but the bending moments of the forces acting from the flow are not balanced, which causes a change in the reference reactions that stimulate the vibration of supporting structures. In this scheme, the central shaft and the traverse connecting the ends of the straight working blades with the shaft resist flow and reduce the energy efficiency of the turbine (Figure 1).

\footnotetext{
* Corresponding author: viprokopiev@ mail.ru
} 

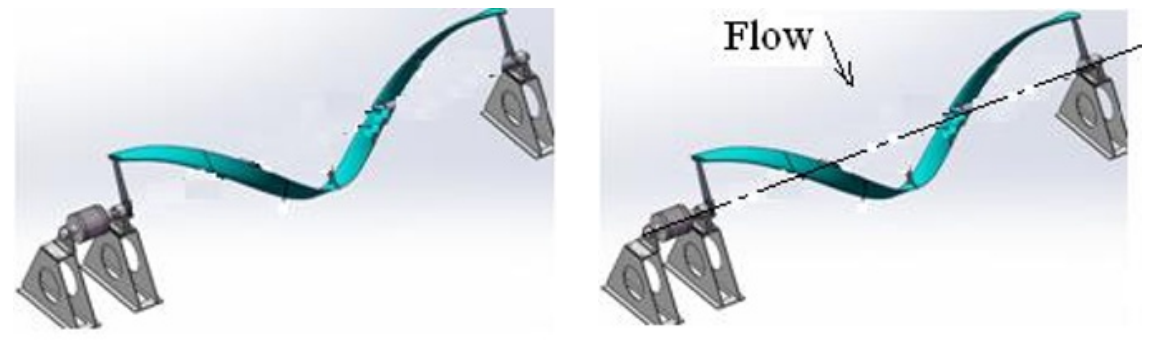

Fig. 1. Single Blade Orthogonal Turbine Scheme.

A construction free from these disadvantages is considered. The turbine contains at least one aerodynamic profile blade curved in a spiral with a constant radius of curvature so that the profile of the blade in each cross section is rotated to the optimum angle relative to the tangent to the circle described by the aerodynamic centre of the profile in this section; the ends of the blade, rotated relative to each other by 360 degrees and, therefore, are in the same position relative to the flow incident on the turbine.

The computational domain (liquid region) was divided into elements in the preprocessor of the ANSYS Workbench software package. The parameters of the calculation grid are shown in Table 1.

Table 1. Characteristics of finite element mesh.

\begin{tabular}{|c|c|c|c|c|}
\hline $\begin{array}{c}\text { Hydraulic } \\
\text { diameter }\end{array}$ & $\begin{array}{c}\text { Reynolds } \\
\text { number }\end{array}$ & $\begin{array}{c}\text { Estimated } \\
\mathbf{Y}+\end{array}$ & $\begin{array}{c}\text { Estimated } \\
\text { distance from } \\
\text { the wall }\end{array}$ & $\begin{array}{c}\text { Mesh thickening in } \\
\text { the longitudinal } \\
\text { direction }\end{array}$ \\
\hline 0.38 & $5.0 \mathrm{e}+4$ & 80 & $1.0 \mathrm{e}-2$ & $1.0 \mathrm{e}-2$ \\
\hline
\end{tabular}

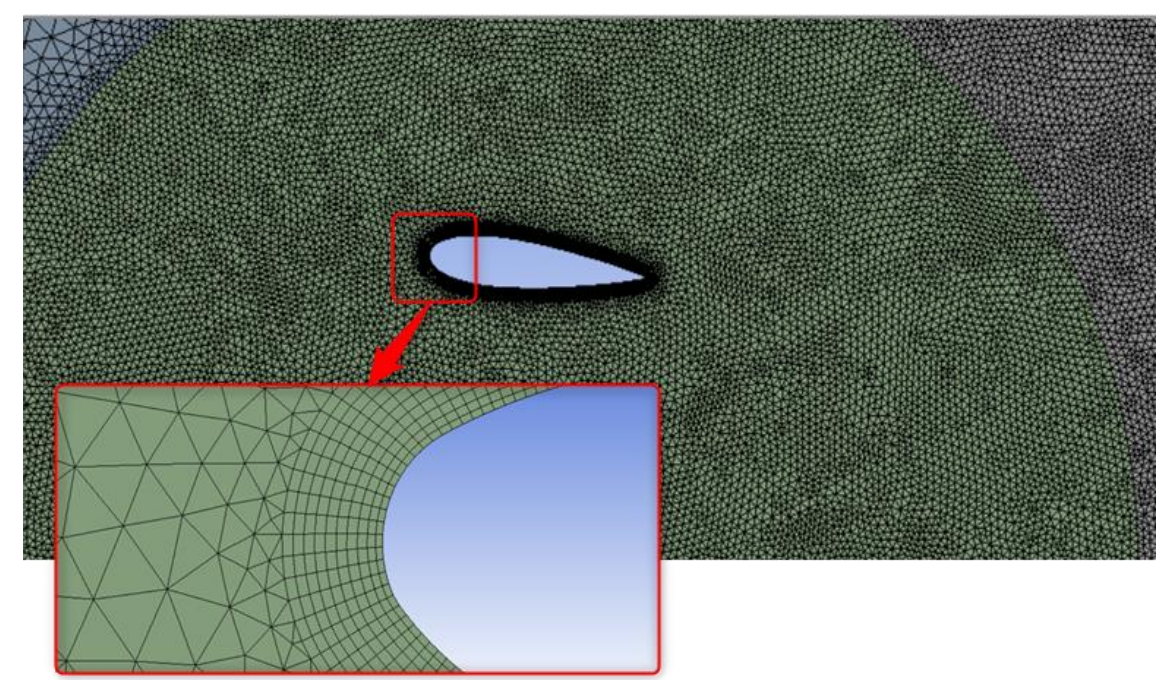

Fig. 2. Finite element mesh.

For aerodynamic calculations, the ANSYS CFX software package was used. The boundary and initial conditions are shown in Figure 3 


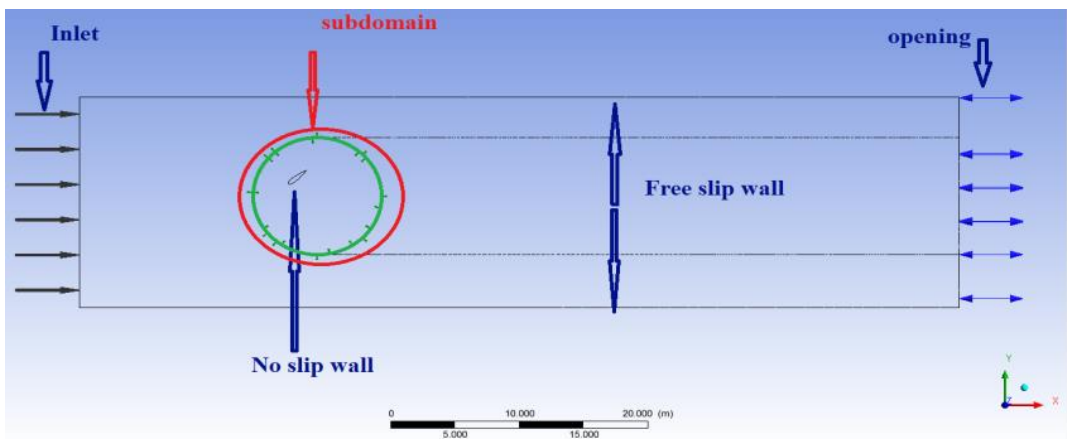

Fig. 3. Boundary and initial conditions.

At the "entrance" (Inlet), the flow velocity was set uniformly distributed over the height and equal to $2 \mathrm{~m} / \mathrm{s}$ and turbulence equal to $7 \%$. At the "Outlet", "soft" boundary conditions of the Opening were set with a relative pressure equal to zero and the same turbulence parameters as at the "inlet". At the lower and upper boundary of the region, the conditions "Walls with sliding" were set (FreeSlipWall, $U=V=W=0 \mathrm{~m} / \mathrm{s}$ ). The blade was given the condition Rigid Body with the possibility of rotation about the $\mathrm{Z}$ axis with a given angular velocity. The cylindrical domain was created as a subdomain with the setting of the recalculation of the mesh of finite elements for the possibility of rotating the blade.

The subdomain, domain interfaces, and paddle must have common air characteristics. This is necessary because the inner cylinder and the rigid body must translate and rotate at the same speed in order to properly isolate the movement. All relative movements between the inner cylinder and the blade will be eliminated, which will lead to the deformation of the zero grid inside the inner cylinder.

Domain interfaces have been edited so as to limit the rotational movement of the mesh surrounding the subdomain. The grid located on the internal cylindrical interface of the domain will have the same physical properties as that of the solid. To set the blade as a solid, the mass per linear meter was used. Based on the thickness of the adopted blade for solving the problem, the mass is $0.135 \mathrm{~kg}$. Since generators of this type cannot start rotation without a third-party pulse, the initial angular velocity was initialized, no other third-party accelerations were initialized.

\section{Results}

Below are the results of aerodynamic calculations. Table 2 shows the maximum (max), minimum (min), average (average) values of lift and drag force, as well as their frequencies. Figure 4 shows a graph of the change in the investigated lift force with respect to time during the rotation of the $\mathrm{F}_{\mathrm{L}}$ blade, and in Figure 5 is a graph of the change in drag force over time $\mathrm{F}_{\mathrm{D}}$.

Table 2. Values and frequencies of the lift forces and forces of drag.

\begin{tabular}{|c|c|c|c|c|}
\hline & $\max , \mathbf{H}$ & $\min , \mathbf{H}$ & average, $\mathbf{H}$ & $\mathbf{f}, \boldsymbol{\Gamma} \mathbf{U}$ \\
\hline$F_{D}$ & 3.785 & -1.666 & 0.182 & 4.327 \\
\hline$F_{L}$ & 4.103 & -1.582 & 0.502 & 4.327 \\
\hline
\end{tabular}




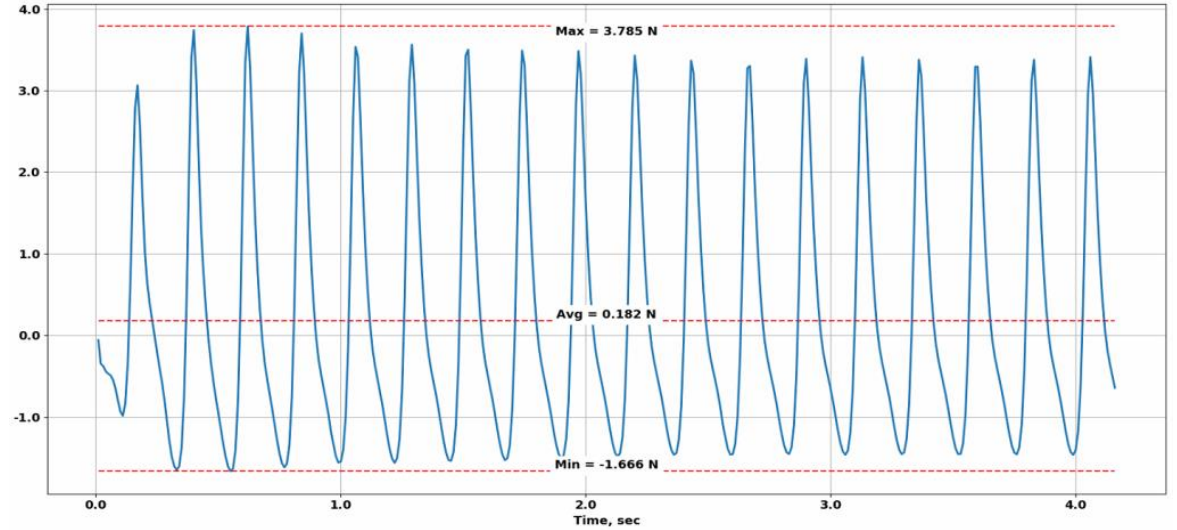

Fig. 4. The graph of the change in the investigated lifting force in time during the rotation of the blade $\mathrm{F}_{\mathrm{L}}, \mathrm{N}$.

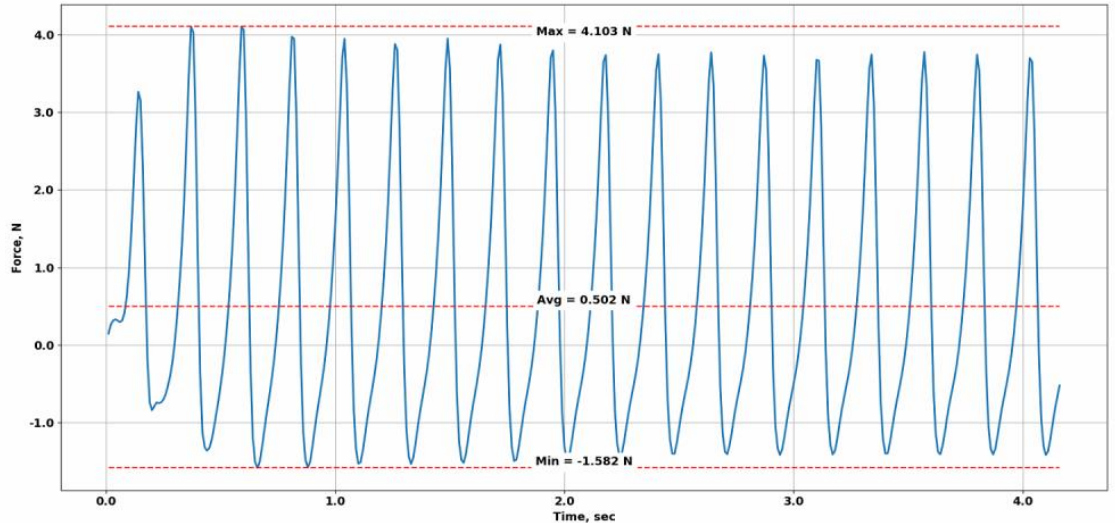

Fig. 5. Graph of changes in drag force in time $F_{D}, N$.

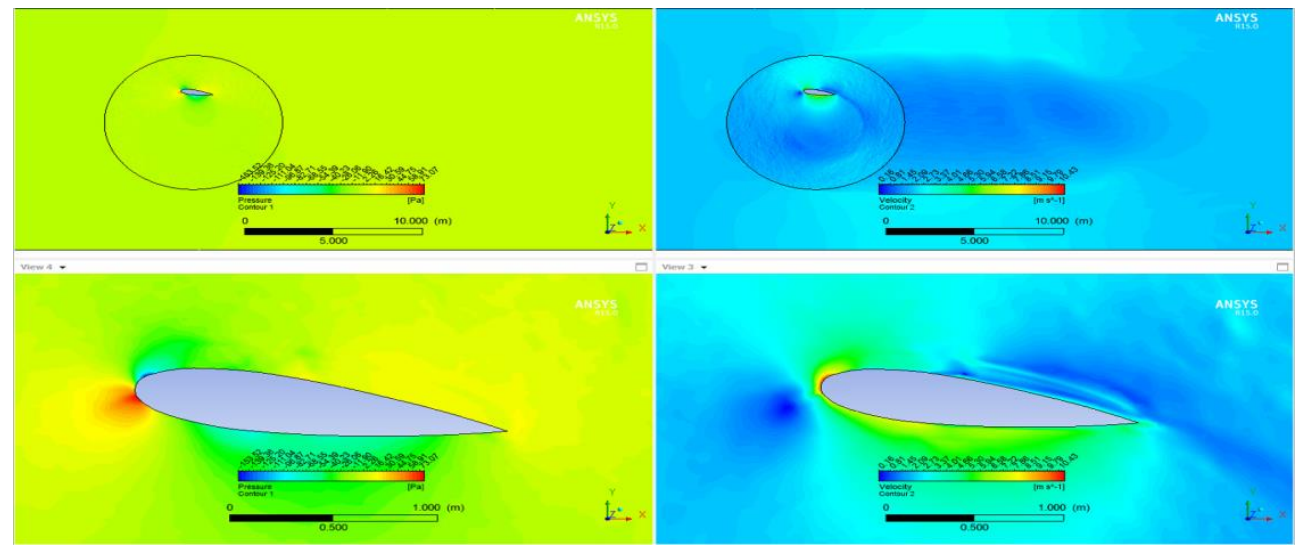

Fig. 6. Fields of velocity and pressures at zero position of the blade (after the full flow of air) 


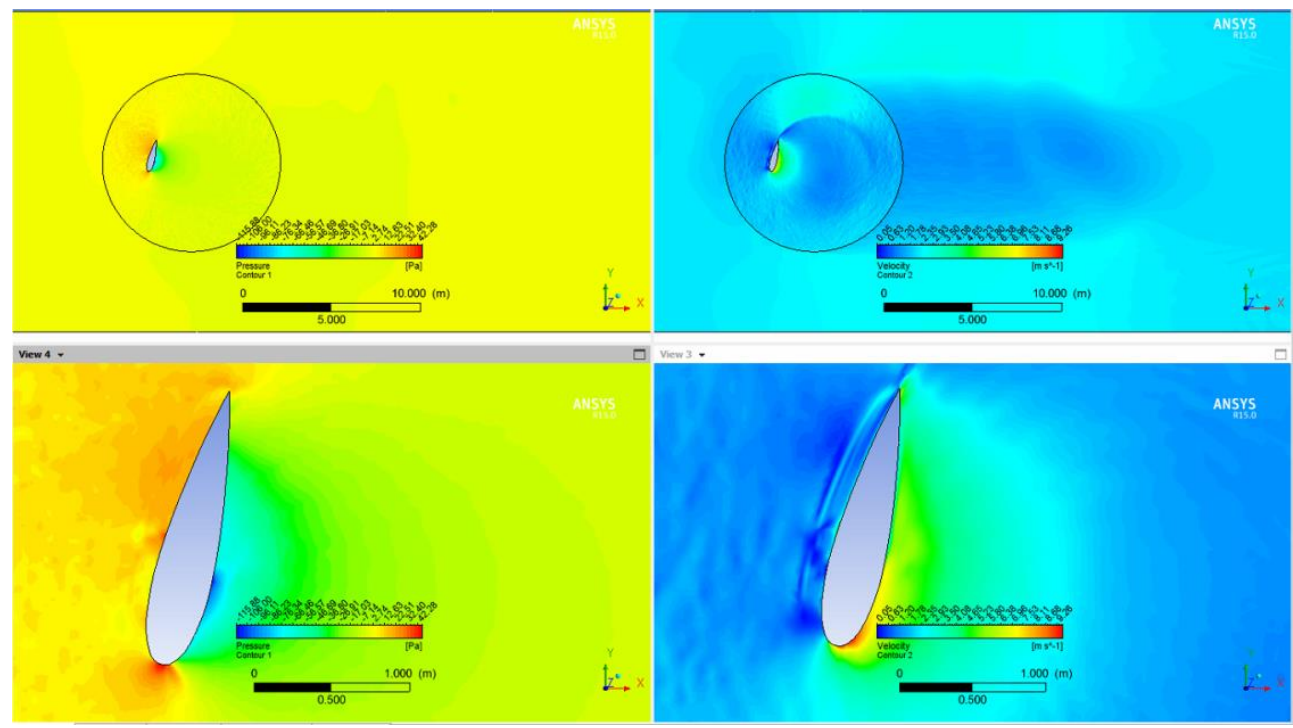

Fig. 6. Fields of velocity and pressures with partial rotation of the blade (after the full flow of air)

\section{Conclusions}

The graphs of the studied forces show the non-stationarity of the process being modeled, each wave corresponds to 1 rotation of the blade.

Frequency analysis shows the coincidence with the frequency of rotation of the blade, while the rotation speed remains constant, the time of 1 revolution of the blade is 1.7 seconds. It can be concluded that the wind generator is rotating in normal mode.

\section{References}

1. A. V. Garbaruk, M. Kh. Sagittarius, M. L. Shur. Simulation of turbulence in the calculations of complex flows. Tutorial (St. Petersburg., 2012)

2. A. E. Lutsky, A. V. Severin, The simplest realization of the method of wall functions. Preprints M. V. Keldysh 038 (2013)

3. O. I. Berg. Principles of construction and elements of control systems for autonomous power supply systems based on renewable energy sources (2015)

4. S. M. Gorlin. Experimental aerodynamics. (High School, 1970)

5. ANSYS, Inc., “ANSYS 15 Help”, 2014.

6. Y. Tominaga, A. Mochida, et al. AIJ guidelines for CFD to pedestrian wind environment around buildings. J. of Wind Eng. and Indust. Aerodynamics. 96, 10-11, (2008) 